\title{
Étude comparative du pouvoir pathogène expérimental de souches d'Acantbamoeba
}

\author{
par P. PERNIN, et A. RIANY \\ Laboratoire de Parasitologie. Faculté de Médecine et de Pharmacie, \\ 8, avenue Rockefeller, 69008 Lyon.
}

RESUME. Nous avons recherché la pathogénicité sur la souris après instillation nasale de 8 souches d'Acanthamoeba isolées de piscines. La souche A1 d'Acanthamoeba culbertsoni a été aussi testée comme souche pathogène de référence.

La virulence est évaluée par un essai de détermination des D.L. 50 pour chaque souche après étude de la variation de la mortalité observée (transformation probit) en fonction de la dose instillée.

On constate que la majorité des souches du genre Acanthamoeba (6 sur 8) isolées à partir du milieu extérieur (piscines) sont capables de provoquer la mort d'un certain pourcentage de souris après instillation nasale. Mais la pathogénicité potentielle ainsi révélée varie dans de grandes proportions (D.L. 50 comprises entre $4.10^{2}$ et $4.10^{5}$ ) d'une souche d'Acanthamoeba à une autre.

Enfin, il ne semble pas exister chez les Acanthamoeba de corrélation entre le pouvoir pathogène observé et l'appartenance à une espèce déterminée.

\section{Comparative study of experimental pathogenicity of Acanthamoeba strains.}

SUMMARY. We have tested pathogenicity on mice after the intranasal instillation of 8 Acanthamoeba strains isolated from swimming-pools. The A1 strain of A. culbertsoni has also been tested as pathogenic reference strain.

Virulence is measured by determination of D.L. 50 for each strain from the study of the variation of mortality in relation to the inoculated dose.

We have established that the most of Acanthamoeba strains (6 on 8) isolated from an external environment (swimming-pools) are able to kill a certain percentage of mice after intranasal instillation. But the potential pathogenicity revealed in this way varies greatly (D.L. 50 included between $4.10^{2}$ and $4.10^{5}$ ) from one strain to another.

Elsewhere there seems to be no correlation among Acanthamoeba between the observed pathogenicity and the fact that it belongs to a particular species.

Accepté le 14 mai 1980. 


\section{Introduction}

Alors que dans le genre Naegleria, seule l'espèce Naegleria fowleri est actuellement reconnue responsable du syndrome de méningoencéphalite amibienne primitive, au sein du genre Acanthamoeba plusieurs espèces, outre A. culbertsoni, présentent un pouvoir pathogène.

Ce sont Culbertson et coll. (1958 et 1959), qui les premiers ont démontré le pouvoir pathogène expérimental du genre Acanthamoeba, à partir de la souche A1 d'Acanthamoeba culbertsoni contaminant une culture cellulaire. Par la suite, ces auteurs rapportent l'existence de souches à virulence plus faible, isolées du milieu extérieur et provoquant une atteinte cérébrale évoluant vers la chronicité (Culbertson et coll., 1966).

Singh et Das en 1970, signalent la virulence d'autres souches d'Acanthamoeba, qu'ils identifient à $A$. rhysodes.

Mais c'est à Kasprzak et Mazur (1972) que revient le mérite d'avoir souligné la proportion élevée de souches d'Acanthamoeba pathogènes isolées à partir du milieu extérieur : sur 24 souches provenant de différents lacs de la région de Posnam, 18 tuent la souris après instillation nasale.

Pour notre part, sur 13 souches d'Acanthamoeba isolées de piscines, 8 ont provoqué la mort des souris par voie nasale (Pernin, 1976).

Si l'ensemble de ces travaux s'accordent à reconnaître l'existence d'un fort pourcentage de souches d'Acanthamoeba pathogènes dans la nature, par contre les difficultés inhérentes aux conditions expérimentales font que jusqu'à présent peu d'études ont été entreprises pour comparer d'une manière précise et quantitative le pouvoir pathogène des différentes souches d'Acanthamoeba entre elles. Quelquefois, les auteurs signalent la virulence mais sans préciser le nombre de trophozoïtes inoculés; le plus souvent, la richesse amibienne de l'inoculum est mentionnée mais aucune D.L. 50 n'est calculée, enfin dans les meilleurs cas un chiffre de D.L. 50 est avancé mais aucun test mathématique ne permet de la vérifier.

C'est pourquoi nous avons entrepris d'évaluer la pathogénicité de différentes souches d'Acanthamoeba isolées de piscines en les comparant à une souche pathogène de référence A. culbertsoni isolée voici 24 ans aux Etats-Unis.

\section{Matériel et méthodes}

\section{A. - Origine des souches.}

Les souches qui ont fait l'objet de cette étude sont au nombre de 9. A part, la souche pathogène de référence $A$. culbertsoni isolée d'une culture cellulaire, toutes les autres souches proviennent de prélèvements effectués dans les piscines de l'agglomération lyonnaise, au cours des années 1974-1975 (Pernin et Riany, 1978) ; elles ont été clonées par nos soins. 
L'identification de ces souches d'Acanthamoeba a été établie par Pussard et Pons (1977) sur les caractères de la morphologie kystique après coloration par la technique de Thierry légèrement modifiée (technique du PATAgr). Elles se répartissent en 6 espèces différentes de la façon suivante :

- A. castellanii : souche L2a.

- A. polyphaga: souche Mer2b.

- A. lugdunensis: souche L3a.

- A. rhysodes: souche R3c.

- A. pustulosa: souche Ger3.

- A. quina : souches L1a,

L3b,

Vil3.

\section{B. - Méthodologie.}

Toutes les souches sont cultivées axéniquement sur milieu liquide C.G.V. (Willaert, 1975) en l'absence d'antibiotiques.

L'obtention d'un nombre suffisant d'amibes nécessite la réalisation de cultures massives obtenues par ensemencement de 150 à $300 \mathrm{ml}$ de milieu C.G.V. réparti en boîtes Falcon (n 3024).

La suspension amibienne de départ est préparée stérilement par centrifugation douce du milieu de culture à $1200 \mathrm{t} / \mathrm{mn}$ pendant $1 \mathrm{mn} \mathrm{30}$, puis décantation du surnageant par aspiration.

L'infestation est réalisée par instillation de $20 \mu \mathrm{l}$ de suspension amibienne à de jeunes souris «swiss » âgées de 3 semaines, préalablement anesthésiées au Nembutal à raison de $50 \mathrm{mg} / \mathrm{kg}$ de poids par voie intra-péritonéale.

L'instillation se fait à l'aide d'une pipette de précision (Gilson, P 20).

Chaque essai de détermination de D.L. 50 repose sur l'instillation de doses différentes d'amibes à 4 lots de 15 souris chacun.

Les dilutions sont réalisées en progression géométrique à partir de la suspension amibienne de départ. La concentration en amibes de chacune des dilutions est vérifiée par double comptage à la cellule de Thoma ou à la cellule de Nageotte.

Les souris sont observées quotidiennement pendant 3 semaines au moins en relevant la mortalité dans chacun des 4 lots. On obtient ainsi pour chaque dose d'amibes instillée un certain pourcentage de décès.

\section{C. - Principe de la détermination des DL 50.}

Nous n'indiquerons ici que les principales étapes de cette détermination (Lazard, 1975). Chaque essai fournit 4 réponses (pourcentages de mortalité $p$ ) correspondant chacune à une certaine dose d'amibes $x$.

Pour linéariser au mieux la courbe de régression de la mortalité en fonction de la dose, les statistiques conseillent de procéder aux changements de variables suivants : 
- Le pourcentage de mortalité observé $p$ est transformé en probit $\mathrm{Y}$ à l'aide d'une table des probits. rithme.

- Enfin, au lieu d'utiliser la dose elle-même, il est préférable d'utiliser son loga-

Après avoir effectué ces changements de variables, on trace alors une première « estimation à vue » de la droite de régression qui va permettre d'utiliser la table des probits et de calculer ensuite l'équation de la droite de régression.

Lors du calcul de l'équation de la droite chaque point est affecté d'un coefficient de pondération $\mathrm{W}$ en fonction de l'importance qui doit lui être attribuée.

$$
\mathrm{W}=n \cdot w
$$

Le poids élémentaire $w$ est donné par les tables, il est multiplié par le nombre d'animaux expérimentés $n$ et donne ainsi le coefficient de pondération W.

L'équation de la droite est de la forme :

$$
\mathrm{Y}-\overline{\mathrm{Y}}=b(x-\bar{x})
$$

Elle passe par le centre de gravité de l'ensemble des $\mathrm{N}$ points qui a pour coordonnées $\overline{\mathrm{Y}}$ et $\bar{x}$ :

$$
\begin{aligned}
& \bar{Y}=\frac{\Sigma W Y}{\Sigma W} \\
& \bar{x}=\frac{\Sigma W x}{\Sigma W}
\end{aligned}
$$

Elle a pour pente :

$$
\begin{aligned}
& b=\frac{\mathrm{SW} x \mathrm{Y}}{\mathrm{SW} x^{2}} \\
& \text { avec }: \mathrm{SW} x \mathrm{Y}=\Sigma \mathrm{W} x \mathrm{Y}-\frac{(\Sigma \mathrm{W} x)(\Sigma \mathrm{WY})}{\Sigma \mathrm{W}} \\
& \text { et } \quad \mathrm{SW} x^{2}=\Sigma \mathrm{W} x^{2}-\frac{(\Sigma \mathrm{W} x)^{2}}{\Sigma \mathrm{W}}
\end{aligned}
$$

On vérifie l'hypothèse de linéarité de la droite de régression calculée par un test X2 à $(L-2)$ d.d.1., $L$ étant le nombre de doses utilisées.

$$
\begin{aligned}
& \mathrm{X}_{(\mathrm{L}-2)}^{2}=\mathrm{SWY}^{2}-\frac{\left(\mathrm{SW} x \mathrm{Y}^{2}\right.}{\mathrm{SW} x^{2}} \\
& \mathrm{SWY}^{2}=\Sigma \mathrm{WY} \mathrm{Y}^{2}-\frac{(\Sigma \mathrm{WY})^{2}}{\Sigma \mathrm{W}}
\end{aligned}
$$

L'hypothèse de linéarité de la droite étant vérifiée, on calcule alors la D.L. 50 par la formule : 


$$
\text { D.L.50 }=\bar{x}+\frac{5-Y}{b}
$$

Pour estimer avec quelle précision a été déterminée cette D.L. 50, il faut calculer sa variance :

$$
\operatorname{Var}\left(\text { D.L. }{ }_{50}\right)=\frac{1}{b^{2}}\left[\frac{1}{\Sigma \mathrm{W}}+\frac{\left(\mathrm{D} . \mathrm{L}_{50}-\bar{x}\right)^{2}}{\mathrm{SW} x^{2}}\right]
$$

La variance permet de préciser l'intervalle de confiance à $95 \%$ de la D.L. 50 :

$$
\text { D.L.50 } \pm 1,96 \sqrt{\text { var.(D.L.50) }}
$$

\section{Résultats}

De nombreux essais ont été effectués. Mais cinq d'entre eux seulement ont permis

\begin{tabular}{|c|c|c|c|c|c|}
\hline Espèce & Souche & \begin{tabular}{|l|}
$\begin{array}{l}\text { Amibes } \\
\text { instillées }\end{array}$ \\
\end{tabular} & \begin{tabular}{|c|}
$\%$ de \\
mortalité
\end{tabular} & D.L.50 & $\begin{array}{l}\text { Limites de } \\
\text { confiance }\end{array}$ \\
\hline \multirow{4}{*}{ A.culbertsoni } & \multirow{4}{*}{$A_{1}$} & 10600 & 100 & \multirow{4}{*}{380} & \multirow{4}{*}{$\begin{array}{c}220 \\
\text { à } \\
650\end{array}$} \\
\hline & & 2120 & 88 & & \\
\hline & & 605 & 68 & & \\
\hline & & 85 & 11 & & \\
\hline \multirow{4}{*}{ A.rhysodes } & \multirow{4}{*}{$\mathrm{R}_{3} \mathrm{C}$} & 3350 & 73 & \multirow{4}{*}{380} & \multirow{4}{*}{$\begin{array}{c}130 \\
\text { à } \\
1110\end{array}$} \\
\hline & & 1120 & 71 & & \\
\hline & & 370 & 46 & & \\
\hline & & 125 & 35 & & \\
\hline \multirow{4}{*}{ A.polyphaga } & \multirow{4}{*}{$\mathrm{Me}_{2} \mathrm{~b}$} & 360000 & 88 & \multirow{4}{*}{$91,4 \times 10^{3}$} & \multirow{4}{*}{$\begin{array}{c}53 \times 10^{3} \\
\text { à } \\
156 \times 10^{3}\end{array}$} \\
\hline & & 75000 & 33 & & \\
\hline & & 17925 & 11 & & \\
\hline & & 4480 & 6 & & \\
\hline \multirow{4}{*}{ A.quina } & \multirow{4}{*}{$\mathrm{Vil}_{3}$} & 376000 & 81 & \multirow{4}{*}{$152,3 \times 10^{3}$} & \multirow{4}{*}{$\begin{array}{c}91,8 \times 10^{3} \\
\text { à } \\
252,6 \times 10^{3}\end{array}$} \\
\hline & & 94000 & 27 & & \\
\hline & & 20000 & 7 & & \\
\hline & & 6800 & 0 & & \\
\hline \multirow{4}{*}{ A.quina } & \multirow{4}{*}{$L_{3} b$} & 448500 & 55.5 & \multirow{4}{*}{$442 \times 10^{3}$} & \multirow{4}{*}{$\begin{array}{l}210 \times 10^{3} \\
\text { à } \\
929 \times 10^{3}\end{array}$} \\
\hline & & 149500 & 12 & & \\
\hline & & 78500 & 6 & & \\
\hline & & 36500 & 0 & & \\
\hline
\end{tabular}
d'aboutir au calcul d'une D.L. 50. Ceci est dû au fait qu'à l'exception d'A. culbertsoni

Tableau I. Détermination des D.L. 50 de cinq souches d'Acanthamoeba 
nous n'avions aucune indication sur le pouvoir pathogène potentiel des souches isolées de piscine.

L'ensemble de nos résultats figurent dans les tableaux I et II. Ils sont accompagnés des 5 droites de régression dont l'équation a pu être déterminée.

Tableau II. Résultats des tests de pathogénicité pour les autres souches

\begin{tabular}{|c|c|c|c|c|c|}
\hline Espèce & Souche & \begin{tabular}{|c|} 
Souris \\
inoculées
\end{tabular} & $\begin{array}{l}\text { Amibes } \\
\text { instillées }\end{array}$ & \begin{tabular}{|c|} 
Décès:Nbre \\
et \\
date en jours
\end{tabular} & $\begin{array}{|cc|}\% & \text { de } \\
\text { mortalité }\end{array}$ \\
\hline \multirow{4}{*}{ A. pustulosa } & \multirow{4}{*}{$\mathrm{Ger}_{3}$} & 15 & 61000 & & 0 \\
\hline & & 16 & 12200 & & 0 \\
\hline & & 16 & 2400 & & 0 \\
\hline & & 15 & 500 & & 0 \\
\hline \multirow{4}{*}{ A.lugdunensis } & \multirow{4}{*}{$\mathrm{L}_{3} \mathrm{a}$} & 15 & 70750 & & 0 \\
\hline & & 15 & 11800 & & 0 \\
\hline & & 14 & 2000 & & 0 \\
\hline & & 16 & 450 & & 0 \\
\hline \multirow{4}{*}{ A. castellanii } & \multirow{4}{*}{$\mathrm{L}_{2} \mathrm{a}$} & 15 & 152000 & $2(8)$ & $13 \%$ \\
\hline & & 15 & 38000 & & 0 \\
\hline & & 16 & 11600 & & 0 \\
\hline & & 16 & 3700 & & 0 \\
\hline \multirow{4}{*}{ A. quina } & \multirow{4}{*}{$\mathrm{L}_{1} \mathrm{a}$} & 14 & 321000 & $\begin{array}{c}2(8), 2(9) \\
3(10), 1(11) \\
1(13), 1(16)\end{array}$ & $71 \%$ \\
\hline & & 16 & 107000 & $\begin{array}{c}2(8) \\
1(16) \\
\end{array}$ & $31 \%$ \\
\hline & & 17 & 34000 & & 0 \\
\hline & & 14 & 13000 & & 0 \\
\hline
\end{tabular}

L'examen de ces tableaux montre que $A$. culbertsoni A1 souche pathogène de référence reste très virulente pour la souris.

Le pouvoir pathogène des souches isolées en piscine varie dans de grandes proportions (de $3,8 \cdot 10^{2}$ pour R $3 c$ à $4,42 \cdot 10^{5}$ pour L 3 ) (fig. 1 et 2 ).

Une de nos souches $A$. rhysodes R $3 c$ apparaît aussi pathogène qu'A. culbertsoni (D.L. 50 identique) avec un intervalle de confiance toutefois plus étendu.

On peut également remarquer (tableau I) que les souches Vil 3 et L $3 b$ morphologiquement identiques et appartenant donc à la même espèce A. quina ont des D.L. 50 qui varient dans des proportions de 1 à 3 (fig. 3 et 4). 


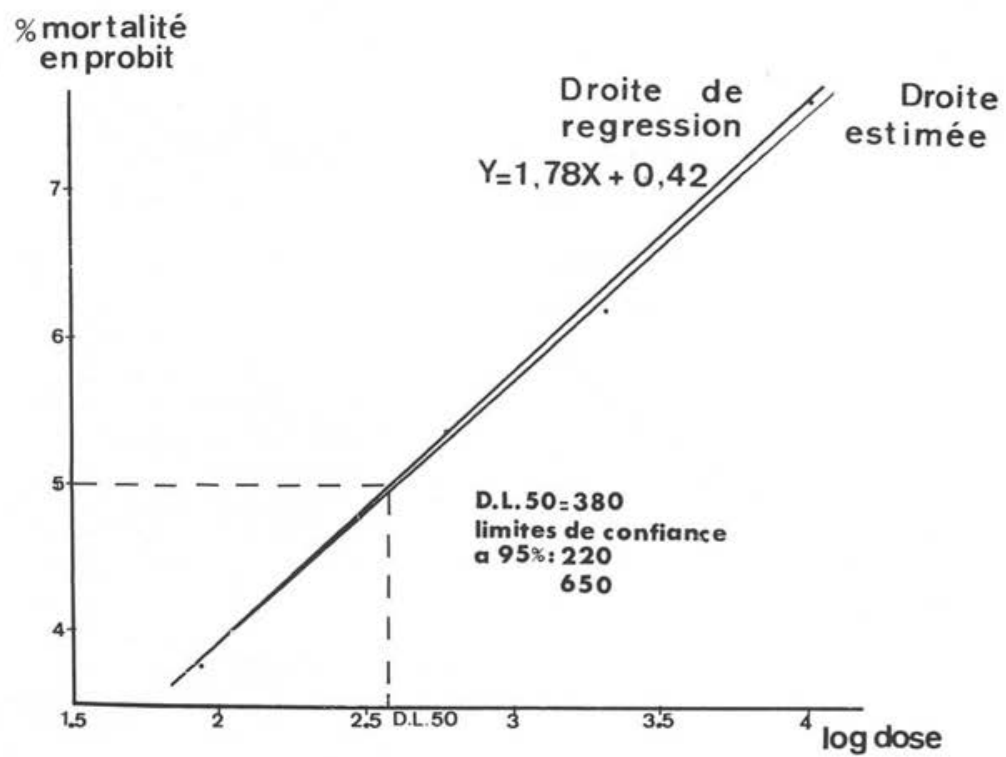

Fig. 1. Détermination de la D.L. 50, A. culbertsoni $\mathrm{A}_{1}$.

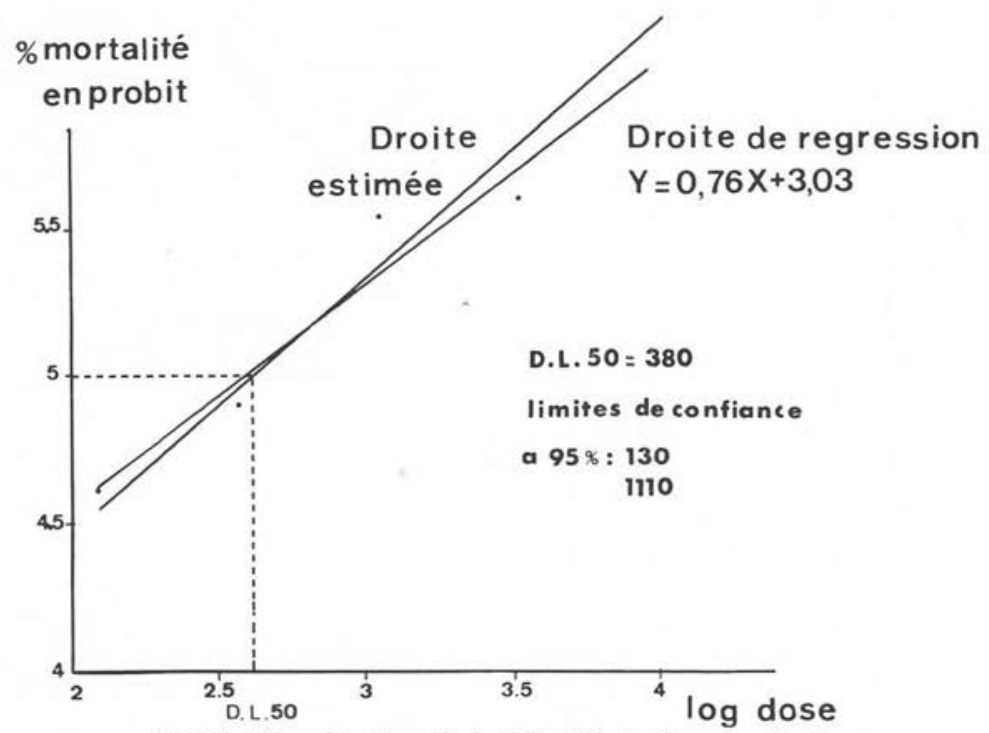

Fig. 2. Détermination de la D.L. 50, A. rhysodes $\mathrm{R}_{3}$ C. 


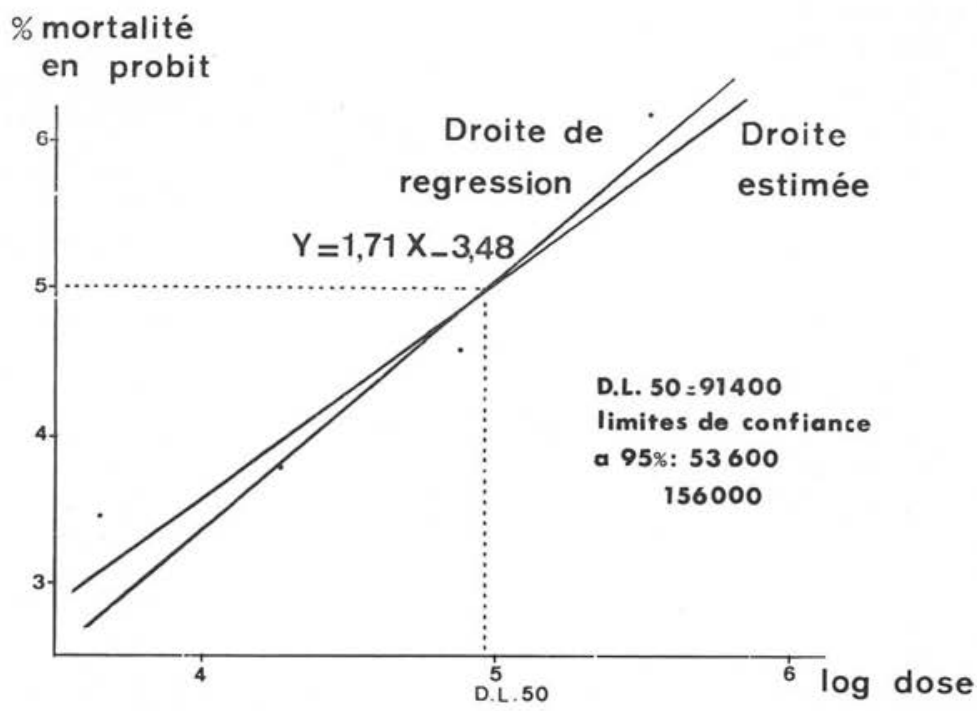

Fig. 3. Détermination de la D.L. 50, A. polyphaga $\mathrm{Mer}_{2} \mathrm{~b}$.

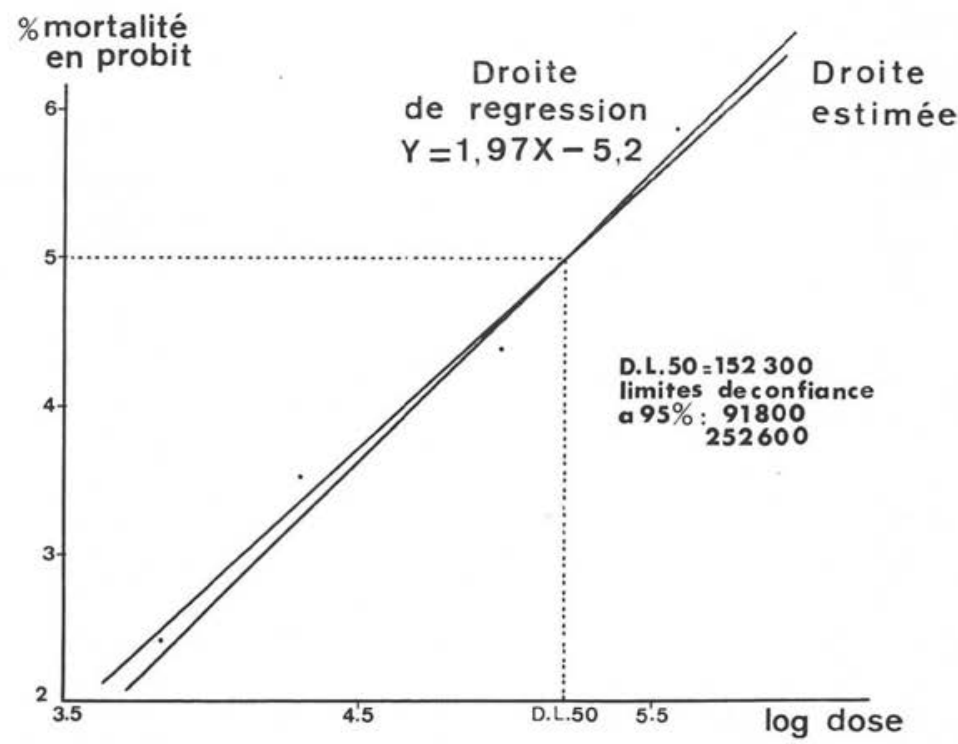

Fig. 4. Détermination de la D.L. 50, A. quina $\mathrm{Vil}_{3}$. 
Pour 4 autres souches (tableau II), il ne nous a pas été possible de déterminer des D.L. 50 en raison des pourcentages de mortalité trop faibles, voire nuls, dus vraisemblablement à un nombre d'amibes instillées insuffisant. Néanmoins, les premiers résultats obtenus permettent déjà d'avoir une idée sur la pathogénicité potentielle de ces souches.

Ainsi, les mortalités observées pour la souche L 1 a se rapprochent de celles obtenues pour la souche Vil 3 , ces 2 souches appartiennent à la même espèce A. quina (fig. 5).

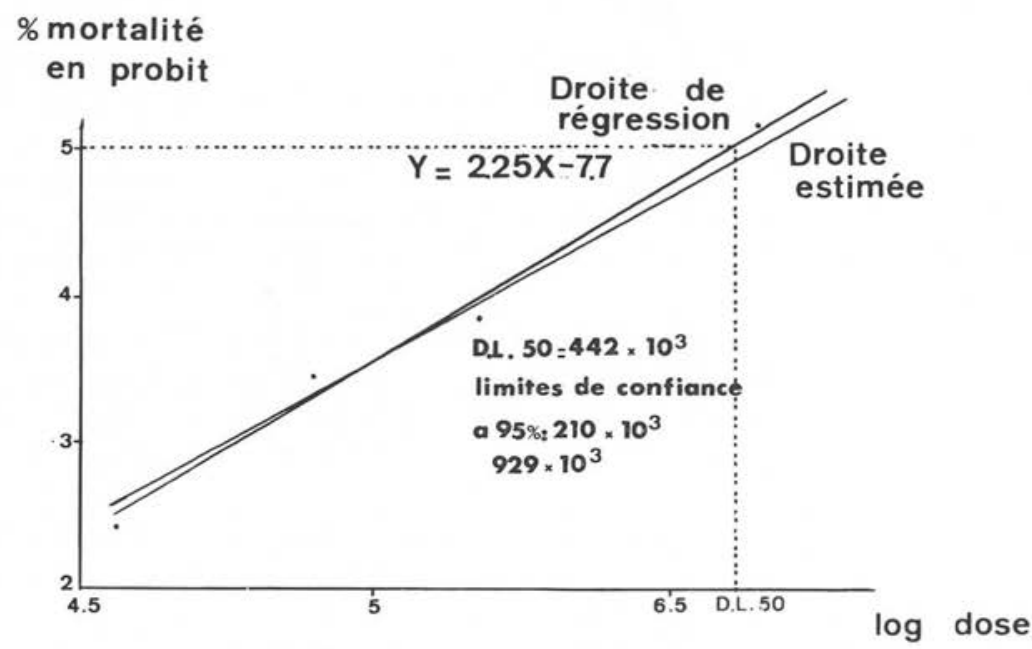

Fig. 5. Détermination de la D.L. 50, A. quina $\mathrm{L}_{3}$ b.

Les souches d'A. pustulosa Ger 3 et d'A. lugdunensis L 3 a n'ont entraîné aucune mortalité aux doses respectives de 61.000 et de 70750 . Des essais supplémentaires nous ont prouvé qu'il faut instiller des doses dépassant 500.000 trophozoïtes pour provoquer la mort des souris. Nous considérons qu'à des concentrations aussi élevées d'amibes dans l'inoculum, il n'est plus possible de connaître avec une précision acceptable le nombre de cellules instillées et les conditions d'une manipulation valable ne sont plus respectées.

\section{Discussion}

Jusqu'à présent seule la pathogénicité des souches hautement virulentes telles que $N$. fowleri ou $A$. culbertsoni a été précisée quantitativement. Les résultats avancés sont-ils encore souvent discordants sans doute en raison des difficultés d'inoculation de la suspension amibienne qui expliquent les divergences constatées chez les différents auteurs quant à la détermination des D.L. 50 de la souche A1 par exemple (Culbertson et coll., 1965 ; Culbertson, 1971 ; Cerva, 1967 ; Stevens et O’Dell, 1974). 
Cerva (loc. cit) montre l'influence considérable de la phase de multiplication logarithmique pour déterminer la D.L. 50. Ces discordances se retrouvent d'ailleurs avec les souches de $N$. fowleri. Ainsi, selon Carter (1970) 39 trophozoïtes de $N$. fowleri inoculés par voie nasale suffisent à tuer la souris alors qu'Anderson et Jamieson (1972) n'obtiennent aucun décès avec 2.000 trophozoïtes d'une souche provenant d'un cas humain. Il leur faut atteindre des doses de 20.000 amibes pour réussir leur implantation chez l'animal.

Nous pensons avoir résolu en partie ces difficultés grâce à l'utilisation d'une micropipette de précision qui élimine l'emploi de la seringue habituelle.

L'utilisation de ce type de pipette présente un double avantage: le volume instillé est délivré avec une exactitude bien supérieure à celle que permet une seringue graduée au $1 / 100^{\mathrm{e}}$ de $\mathrm{ml}$; d'autre part, selon le principe des micropipettes, le volume constant est aspiré avant chaque instillation : il devient dès lors possible de renouveler l'homogénéisation de la suspension amibienne entre chaque animal d'un même lot expérimental. On surmonte ainsi les variations liées à la sédimentation des amibes dans le corps de la seringue.

Une deuxième difficulté qui peut faire varier considérablement le nombre d'amibes instillées provient du risque de refoulement par éternuement ou de régurgitation d'une partie du volume administré. L'obtention d'une anesthésie assez profonde au Nembutal permet d'éviter en principe les réactions de rejet et d'assurer une inhalation complète des $20 \mu \mathrm{l}$.

Nous retrouvons dans ces conditions d'expérimentation une pathogénicité élevée (D.L. $50: 380$ ) pour A. culbertsoni souche A1. Ce chiffre parait compatible vu les difficultés expérimentales, avec ceux rapportés par Culbertson (1971), d'une part (D.L. $50: 20$ à 50) et par Cerva (1967) d'autre part (D.L. $50: 30$ à 300).

A. culbertsoni isolée voici plus de 20 ans aux U.S.A. et entretenue depuis en culture a donc conservé intégralement sa virulence. La persistance d'un pouvoir pathogène élevé chez A. culbertsoni se retrouve également dans les travaux de Willaert (1975).

La pathogénicité chez les souches d'Acanthamoeba apparaît donc comme un caractère plus stable que chez Naegleria fowleri pour laquelle on assiste à une diminution très nette de la virulence au cours des repiquages successifs tout au moins pour les souches provenant du milieu extérieur (De Jonckheere, 1979).

On constate que la plupart des souches d'Acanthamoeba que nous avons testées sont capables d'entraîner la mort d'un certain pourcentage d'animaux après instillation nasale si l'on prend soin de travailler avec une dose suffisante : ainsi au cours des essais de détermination de D.L. 50 sur 8 souches isolées de piscines, 6 ont pu provoquer la mort des animaux. Si la plupart du temps ces souches apparaissent comme faiblement pathogènes avec des D.L. 50 comprises entre 100.000 et 200.000 amibes, une de nos propres souches $A$. rhysodes $\mathrm{R} 3 c$ doit néanmoins être rangée dans la catégorie des espèces à haut risque en raison de sa D.L. 50. Le pouvoir pathogène de cette espèce a été signalé pour la première fois par Singh et Das (1970) qui rapportent une mortalité de $100 \%$ pour 20.000 trophozoïtes sans toutefois indiquer aucune différence dans la virulence de 12 souches testées. 
D'après nos résultats, il n'existe pas de corrélation au sein du genre Acanthamoeba entre le degré de pathogénicité d'une souche et son appartenance à une espèce déterminée: ainsi $A$. rhysodes (souche $\mathrm{R} 3 c$ ) présente une pathogénicité comparable à A. culbertsoni. Cette constatation diffère de ce qui a été établi pour le genre Naegleria où Willaert (1975) a démontré que toutes les souches pathogènes appartiennent à une seule et même espèce définie antigéniquement : Naegleria fowleri.

Il existe d'autre part, une variation intraspécifique de la virulence comme c'est le cas pour les souches Vil 3 et L $3 b$ qui présentent des D.L. 50 différentes bien qu'elles appartiennent morphologiquement à la même espèce $A$. quina. De même, le pouvoir pathogène d'A. castellanii paraît assez variable d'une souche à une autre. Alors que notre clone L $2 a$ paraît peu virulent (13\% de mortalité pour 152.000 amibes), Willaert (1975) obtient respectivement 60 et $100 \%$ de mortalité avec les souches 1930 et Rus 22 d'Acanthamoeba castellanii après instillation d'environ 5.000 trophozoïtes.

En conclusion, ce travail nous rappelle que chez les amibes de l'eau et du sol, la capacité de passer d'un état de vie libre dans le milieu extérieur à un mode de vie parasitaire n'est pas un caractère exclusif de l'espèce Naegleria fowleri. La possibilité d'adaptation au parasitisme est réalisable avec de nombreuses souches d'Acanthamoeba isolées du milieu extérieur (plus précisément ici de piscines) en dehors même de l'espèce $A$. culbertsoni.

Mais dans l'état actuel de nos connaissances, il paraît difficile de relier l'existence d'un pouvoir pathogène chez les Acanthamoeba à un critère quelconque, fusse-t-il morphologique, biochimique, ou antigénique.

\section{Bibliographie}

Anderson K., Jamieson A. : Agglutination test for the investigation of the genus Naegleria. Pathology, 1972, 4, 273-278.

Carter R.F.: Description of a Naegleria isolated from two cases of primary amoebic meningo encephalitis, and of the experimental pathological changes induced by it. J. Pathol., 1970, 100, 217-244.

Cerva L.: Intranasal, intrapulmonary and intracardial inoculation of experimental animals with Hartmannella castellanii. Folia Parasitol., 1967, 14, 207-215.

Culbertson C. G., Smith J. W., Minner J.R.: Acanthamoeba : observations on animal pathogenicity. Science, 1958, 127, 1506.

Culbertson C. G., Smith J.W., Cohen H.K., Minner J.R.: Experimental infection of mice and monkeys by Acanthamoeba. Am. J. Pathol., 1959, 35, 185-197.

Culbertson C. G., Holmes D. H., Overton W. M. : Hartmannella castellanii (Acanthamoeba sp.). Preliminary report on experimental chemotherapy. Am. J. Clin. Path., 1965, 43, 361-364.

Culbertson C. G., Ensminger P.W., Overton W. M.: Hartmannella (Acanthamoeba). Experimental chronic, granulomatous brain infections produced by new isolates of low virulence. Am. J. Clin. Pathol., 1966, 46, 305-314.

Culbertson C. G.: The pathogenicity of soil amoebas. Annual. Rev. Microbiol., 1971, 25, 231-254.

De Jonckheere J.: Difference in virulence of Naegleria fowleri. Path. Biol. 1979, 27, 453-458. 
Kasprzak W., Mazur T. : Free-living amoebae isolated from waters frequented by people in the vicinity of Poznan, Poland. Experimental studies in mice of the pathogenicity of the isolates. Z. Tropen. Med. Parasit., 23, 391-398.

Lazard P. : Les essais biologiques. Cours du C.E.S.A.M. Université Pierre et Marie Curie, Paris, 1975.

Pernin P. : Recherche et essai d'identification par microscopie en contraste de phase des amibes libres des piscines lyonnaises. Etude de la pathogénicité des souches de Naegleria sp. et d'Acanthamoeba sp. isolées. Thèse d'Etat de Pharmacie, 1976, $\mathrm{n}^{\circ} 127,257$ p. Université Claude Bernard, Lyon I.

Pernin P., Riany A. : Etude sur la présence d'amibes libres » dans les eaux des piscines lyonnaises. Ann. Parasitol., Hum. Comp., 1978, 53, 333-343.

Pussard M., Pons R. : Morphologie de la paroi kystique et taxonomie du genre Acanthamoeba (Protozoa, Amoebida). Protistologica, 1977, 13, 557-598.

Singh B.N., Das S. R. : Studies on pathogenic and non pathogenic small free-living amoebae and the bearing of nuclear division on the classification of the order Amoebida. Phil. Trans. Roy. Soc. London, 1970, 259, 435-476.

Stevens A. R., O'Dell W.D.: In vitro growth and virulence of Acanthamoeba. J. Parasitol., 1974, $60,884-885$.

Willaert E. : Etude immunotaxonomique des genres Naegleria et Acanthamoeba (Protozoa, Amoebida). Thèse Doctorat Sciences, 1975, Lille, 239 p. 